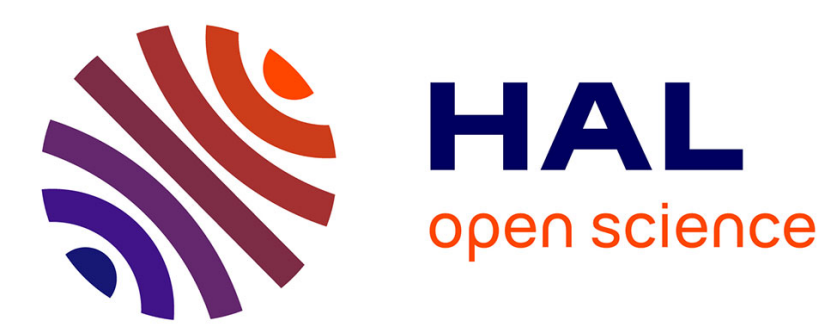

\title{
Structural Sensitivity of Neural and Genetic Networks
}

\author{
Hedi Ben-Amor, Jacques Demongeot, Sylvain Sené
}

\section{To cite this version:}

Hedi Ben-Amor, Jacques Demongeot, Sylvain Sené. Structural Sensitivity of Neural and Genetic Networks. MICAI 2008: Advances in Artificial Intelligence, 7th Mexican International Conference on Artificial Intelligence, Oct 2008, Mexico, Mexico. pp.937-986, 10.1007/978-3-540-88636-5_92 . hal-00346566

\section{HAL Id: hal-00346566 https://hal.science/hal-00346566}

Submitted on 12 Dec 2008

HAL is a multi-disciplinary open access archive for the deposit and dissemination of scientific research documents, whether they are published or not. The documents may come from teaching and research institutions in France or abroad, or from public or private research centers.
L'archive ouverte pluridisciplinaire HAL, est destinée au dépôt et à la diffusion de documents scientifiques de niveau recherche, publiés ou non, émanant des établissements d'enseignement et de recherche français ou étrangers, des laboratoires publics ou privés. 


\title{
Structural Sensitivity of Neural and Genetic Networks
}

\author{
Hedi Ben Amor ${ }^{1}$, Jacques Demongeot ${ }^{1}$, and Sylvain Sené ${ }^{1,2}$ \\ 1 TIMC-IMAG, Faculty of medecine, University Joseph Fourier of Grenoble, \\ 38706 La Tronche, France \\ 2 IXXI, Rhône-Alpes Complex Systems Institute, 5 rue du Vercors, \\ 69007 Lyon, France
}

\begin{abstract}
This paper aims at giving new results on the structural sensitivity of biological networks represented by threshold Boolean networks and ruled by Hopfield-like evolution laws classically used in the context of neural and genetic networks. Indeed, the objective is to present how certain changes and/or perturbations in such networks can modify significantly their asymptotic behaviour. More precisely, this work has been focused on three different kinds of what we think to be relevant in the biological area of robustness (in both theoretical and applied frameworks): the boundary sensitivity (external fields, hormone flows, ...), the state sensitivity (axonal or somatic modulations, microRNAs actions, ...) and the updating sensitivity.
\end{abstract}

\section{Introduction}

Robustness studies in biological networks constitute now a real challenge on both views $i$ ) of the evolution $[1,2]$ : what kind of structures insensitive to environmental perturbations have been selected? and $i i$ ) of the function $[3,4]$ : which type of critical part of a regulatory network is concerned by its function? The first point concerns the speciation with inheritance of interaction architectures having already shown their robustness and the second concerns the differentiation, i.e., the ability to create new functions, corresponding to new dynamical regimes of the network fulfilling new functions by optimising new local cost functionals.

A evolutionary view on inheritance of regulatory networks leads to build phylogenetic trees of networks by using adequate distances between their interaction graphs in order to calculate barycenters of clusters and extract putative ancestors. A way to validate these trees could be to follow the increasing robustness of their leaves and a good characterization of this robustness could be a score incorporating three kinds of sensitivity: boundary sensitivity (to external chemico-physical fields like substrate input or to external controllers like hormonal morphogenetic or growth factors), state sensitivity (to endogeneous action like neuronal axonal modulations of microRNAs inhibition) and updating sensitivity (to changes in dynamical behaviours induced by the mode of updating, parallel, sequential, etc.). Each sensitivity can be quantified by the mean probability to change of stability basin under the corresponding perturbation $[5,6]$. 
So, in this paper, after having presented the major useful definitions in Section 2, we give in Section 3 new results on the influence of boundary conditions in stochastic Hopfield-like networks. More precisely, we focus on the differences between the impact of extremal and "quincuncial" boundaries on theoretical networks, i.e., lattices on $\mathbb{Z Z}^{2}$. In Section 4 , we consider real regulatory networks, one devoted to the control of hair morphogenesis in mice and the second to the cell cycle control in superior eukaryotes, and we show in which way the addition of critical nodes in these networks can perturb their attractors.

\section{Preliminary definitions}

We consider in the following Hopfield-like networks [7]. More precisely, we denote by $R$ a connected biological network of $N$ nodes having two possible activity states such that $\sigma_{i}(t)=0$ (resp. 1) if node $i$ is inactive (resp. active) at time $t$. We call $\sigma(t)=\left(\sigma_{i}(t)\right)_{i \in R} \in \Omega=\{0,1\}^{N}$ the configuration of the network at time $t$, where $\Omega$ is the set of all possible configurations of $R$. Such networks can be represented by oriented graphs in which vertices correspond to the nodes and the directed edges to the interactions between these nodes. Each interaction is characterised by a potential which gives the weight of the action that the source node gets on the destination node. Being given such an arbitrary network $R$, we associate to it an interaction matrix $W_{N \times N}$ whose coefficient $w_{i j}$ corresponds to the interaction potential that node $j$ exerts on node $i$. More precisely, the coefficient $w_{i j}$ can be positive or negative depending on the fact that node $j$ tends to respectively activate or inhibit node $i$ and is null if $j$ has no influence on $i$.

In these networks, the centre is defined by the set of nodes whose eccentricity (i.e., maximal length of the shortest paths to reach from the different nodes all the others) is minimal and the boundary by the set of Gardens of Eden (i.e., nodes whose inner degree is null) whose distance to the center is greater than the minimal distance from the center to all its accessible pendant edges (i.e., nodes whose outer degree is null). Note that if there is not such pendant node, the boundary of the network is the set of all Gardens of Eden.

In the sequel of this article, we will focus on two different kinds of Hopfieldlike neural networks whose evolution is ruled either by a deterministic law or by a stochastic law. Let us now present these two evolution laws.

Let $\mathcal{N}_{i}$ be the neighbourhood of node $i$, i.e., the set of nodes $j$ 's having an influence on $i$. More formally, $j \in \mathcal{N}_{i} \Leftrightarrow w_{i j} \neq 0$. Let us also denote by $H\left(\sigma_{i}(t)\right)$ the interaction potential of node $i$ at time $t . H\left(\sigma_{i}(t)\right)$ is then defined by:

$$
H\left(\sigma_{i}(t)\right)=\sum_{j \in \mathcal{N}_{i}} w_{i j} \cdot \sigma_{j}(t)-\theta_{i}
$$

where $\theta_{i}$ is the activation threshold of node $i$, i.e., the quantity of interaction potential that has to be overtaken in order to node $i$ becomes (or stays) active. 
From this interaction potential, we define respectively as follows the deterministic (rule 1) and the stochastic (rule 2) Hopfield-like evolution rules:

$$
\sigma_{i}(t+1)=\mathcal{H}\left(H\left(\sigma_{i}(t)\right)\right)
$$

where $\mathcal{H}$ is the Heaviside (or sign-step) function such that:

$$
\mathcal{H}(x)= \begin{cases}0 & x \leq 0 \\ 1 & \text { otherwise }\end{cases}
$$

and

$$
P\left(\sigma_{i}(t+1)=\alpha \mid \sigma_{j}(t), j \in \mathcal{N}_{i}\right)=\frac{e^{\alpha \cdot H\left(\sigma_{i}(t)\right) / T}}{1+e^{H\left(\sigma_{i}(t)\right) / T}}
$$

where $T$ is the temperature of the system.

Let us remark that the temperature allows to render the system more or less probabilistic. Indeed, when the temperature tends to 0 , the stochastic rule is equivalent to the deterministic one and, when the temperature tends to infinity, the probability for the state of a node to be equal to 1 is $\frac{1}{2}$.

\section{Extremal and "quincuncial" boundaries in $2 \mathrm{D}$ theoretical networks}

The comprehension of the behaviour of real biological regulatory networks is a difficult problem that researchers, from many different disciplines, are now more and more to study. Some works have highlighted the particular topology of biological networks such as neural and genetic regulation networks [8,9]. Nevertheless, their behaviour can be sometimes better understood by studying more theoretical networks such as the ones of cellular automata [10]. However, most researches have been focused on the comprehension of emergent phenomena in infinite and periodic (toric) cellular automata [11,12], which could be criticised from a biological point of view. Indeed, living systems do not develop infinitely and each of their components, e.g., a cell, is confined in a large but finite space. That is why, in this part, we decide to focus on finite systems, with fixed boundaries surrounding them. More precisely, in [13], the authors present simulation results highlighting that the influence of fixed boundary conditions can lead to the emergence of phase transitions in the asymptotic behaviour of stochastic Hopfield-like networks. In this section, with the same method, we decide to go further and show that the nature of the deployed fixed boundary conditions can affect quantitatively these phase transitions.

\subsection{Definitions and simulation protocol}

The networks on which we focus here are represented by two-dimensional square lattices whose elements are located at vertices of $\mathbb{Z}^{2}$ and are governed by the stochastic evolution law. More formally, if we denote such a network 
composed by $N$ nodes by $R$, it is defined by: $R=([-k, \ldots, k] \times[-k, \ldots, k]) \cap \mathbb{Z}^{2}$ and $N=(2 \cdot k+1)^{2}$. Furthermore, the neighbourhood of a node $i$ belonging to $R$ is the set of five nodes composed by $i$ itself and its four nearest neighbours $j_{1}, j_{2}, j_{3}$, and $j_{4}$ such that $\forall k \in\{1, \ldots, 4\}, d\left(i, j_{k}\right)=1$. Likewise in [13], we define the boundary of such a network by the set of nodes of degree (i.e., number of neighbours in $R$ ) less or equal than 4 . In the same way, we define the centre $c$ of the network by the node being at equal distance of the four corners of the lattice. Let us remark that these definitions of boundary and centre can be generalised to arbitrary oriented graphs by using the definition of graph eccentricity as in $[5,6]$ and that they are also in respect with the ones given in Section 2. As it has been introduced above, the objective is to compare the effects on the asymptotic behaviour of such networks induced by fixed boundary conditions of different nature. In order to have more chance to obtain significant results on this subject, we study the two farthest kinds of boundary conditions in the spectrum of possibles, i.e., the extremal and the quincuncial ones. By stretching out the boundary conditions by beginning from the upper left corner of the lattice and going clockwise, we can represent them by vectors of size $4 \cdot \sqrt{N}-4$. The extremal ones (denoted by $B_{e}$ ) are then distinguished in two types: $B_{e}^{(0)}=$ $\{0,0, \ldots, 0\}$ and $B_{e}^{(1)}=\{1,1, \ldots, 1\}$ as well as the quincuncial ones (denoted by $\left.B_{q}\right): B_{q}^{(01)}=\{0,1,0,1, \ldots, 0,1\}$ and $B_{q}^{(10)}=\{1,0,1,0, \ldots, 1,0\}$.

We define the activity of a node $i$ by the number of time iterations at which $i$ is active. The measure of the influence of fixed boundary conditions is based on the computation of the asymptotic activity of the centre of the network. Let us denote by $T_{t}$ the transient time during which the system evolves to reach its asymptotic behaviour and by $T_{s}$ the sampling time during which the activity of the centre is computed. Thus, the central activity of a network is defined by: $S=\sum_{t \in T_{s}} \sigma_{c}(t)$ and corresponds consequently to the number of sampling time iterations during which the center $c$ has been observed active. Depending on the chosen fixed boundary conditions, we can easily obtain $S^{(0)}, S^{(1)}, S^{(01)}$ and $S^{(10)}$. The measures (computed thanks to Monte-Carlo simulations) are then respectively for extremal and quincuncial boundaries: $S_{e}=\frac{\left|S^{(0)}-S^{(1)}\right|}{\left|T_{s}\right|}$ and $S_{q}=\frac{\left|S^{(01)}-S^{(10)}\right|}{\left|T_{s}\right|}$. It has been shown in [13] that, in certain domains of the parameters $u_{0}=\frac{w_{i i}}{T}$ and $u_{1}=\frac{w_{i j}}{T}$ and for networks sizes of different order of magnitude, the influence of fixed boundary conditions is significant and leads to the emergence of phase transitions. In this work, we focus on homogeneous (isotropic and translation invariant) attractive $\left(u_{1}>0\right)$ and repulsive $\left(u_{1}<0\right)$ networks. Since the existence of phase transitions has been highlighted in [13], we simulate the asymptotic behaviour of small 2 D networks of size $11 \times 11$ and execute a Monte-Carlo algorithm on 500 different initial configurations of density 0.5 , i.e., the probability for a node to be activated at time $t=0$ is equal to $\frac{1}{2}$.

\subsection{Results}

Let us now present some new results concerning the correlations between the influence and the nature of fixed boundary conditions. We emphasise the higher 
influence of extremal boundaries in attractive Hopfield-like networks before highlighting the significant impact of quincuncial boundaries in repulsive networks. To do that, we focus on the measures $S_{e}$ and $S_{q}$ defined above and on the specific probabilities to observe (in the phase transitions domains of parameters $u_{0}$ and $u_{1}$ ) the following extremal and quincuncial patterns over sampling time on the central square of size $3 \times 3$ :

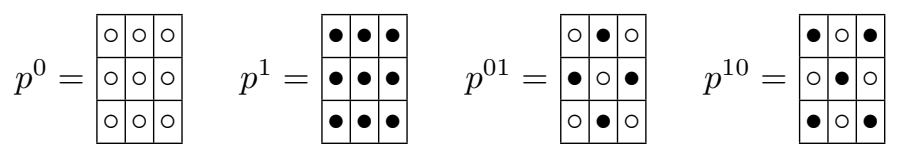

In particular, we emphasise that the computed measures $S_{e}$ and $S_{q}$ are correlated to these probabilities that give a statistical explanation of the quantitative differences observed. We define by $P_{0}^{0}$ (resp. $P_{1}^{0}, P_{01}^{0}, P_{10}^{0}$ ) the probability to observe the pattern $p^{0}$ when boundaries states are fixed to 0 (resp. 1, the quincunx 01 and the quincunx 10). In the same way, we define by $P_{x}^{1}\left(\right.$ resp. $\left.P_{x}^{01}, P_{x}^{10}\right)$ the probability to observe $p^{1}$ (resp. $p^{01}, p^{10}$ ) where $x$ can be 1, 01 or 10 . The probability measure, depending on the probabilities to observe specific patterns, relevant to establish the correlation introduced above, is the minimal proportion $\mathcal{P}$ of sampling time iterations on which different patterns (specific or not) can be observed. Indeed, observing different patterns is a necessary condition to observe values variations of the measures $S^{(0)}$ and $S^{(1)}$ or $S^{(01)}$ and $S^{(10)}$. In the logic terminology, the event $\mathcal{E}$ of probability $\mathcal{P}$ is defined in the extremal (resp. quincuncial) boundaries case by $\mathcal{E}_{e}=\neg\left(\left(E_{0}^{0} \wedge E_{1}^{0}\right) \vee\left(E_{0}^{1} \wedge E_{1}^{1}\right) \vee\left(E_{0}^{01} \wedge E_{1}^{01}\right) \vee\left(E_{0}^{10} \wedge E_{1}^{10}\right)\right)$ (resp. $\left.\mathcal{E}_{q}=\neg\left(\left(E_{01}^{0} \wedge E_{10}^{0}\right) \vee\left(E_{01}^{1} \wedge E_{10}^{1}\right) \vee\left(E_{01}^{01} \wedge E_{10}^{01}\right) \vee\left(E_{01}^{10} \wedge E_{10}^{10}\right)\right)\right)$ where $E_{x}^{p}$ denotes the event of probability $P_{x}^{p}$ to observe the pattern $p$ with the boundary $x$. Since we want to obtain probabilities lower bounds, the probabilities $\mathcal{P}_{e}$ and $\mathcal{P}_{q}$ are defined by complement to 1 of the maximal probabilities to have a specific pattern and we have the following lower bounds:

$$
\begin{gathered}
\mathcal{P}_{e} \geq 1-\left[\sum_{x=0, y=1} \min \left(P_{x}^{0}, P_{y}^{0}\right)+\ldots+\min \left(P_{x}^{10}, P_{y}^{10}\right)\right] \\
\mathcal{P}_{q} \geq 1-\left[\sum_{x=01, y=10} \min \left(P_{x}^{0}, P_{y}^{0}\right)+\ldots+\min \left(P_{x}^{10}, P_{y}^{10}\right)\right]
\end{gathered}
$$

Notice that the standard deviations of the $\mathcal{P}$ 's are the sum of the four maximal standard deviations of the couples $\left(P_{x}^{p}, P_{y}^{p}\right)$ where $p=0,1,01,10$ and that for $(x, y)=(0,1)$ or $(01,10)$.

Let us first focus on attractive networks. The figures presented in Table 1 show that the influences exerted by extremal and quincuncial fixed boundary conditions are qualitatively very close. Indeed, the two kinds of boundary conditions bring to the emergence on the straight line $u_{0}+2 \cdot u_{1}=0$ of a phase transition that has been studied in [13]. However, the emergence of phase transition is quantitatively significantly more important with extremal than with quincuncial boundary conditions, which can be explained by the probabilities introduced above and presented in the second part of Table 1. Before studying 


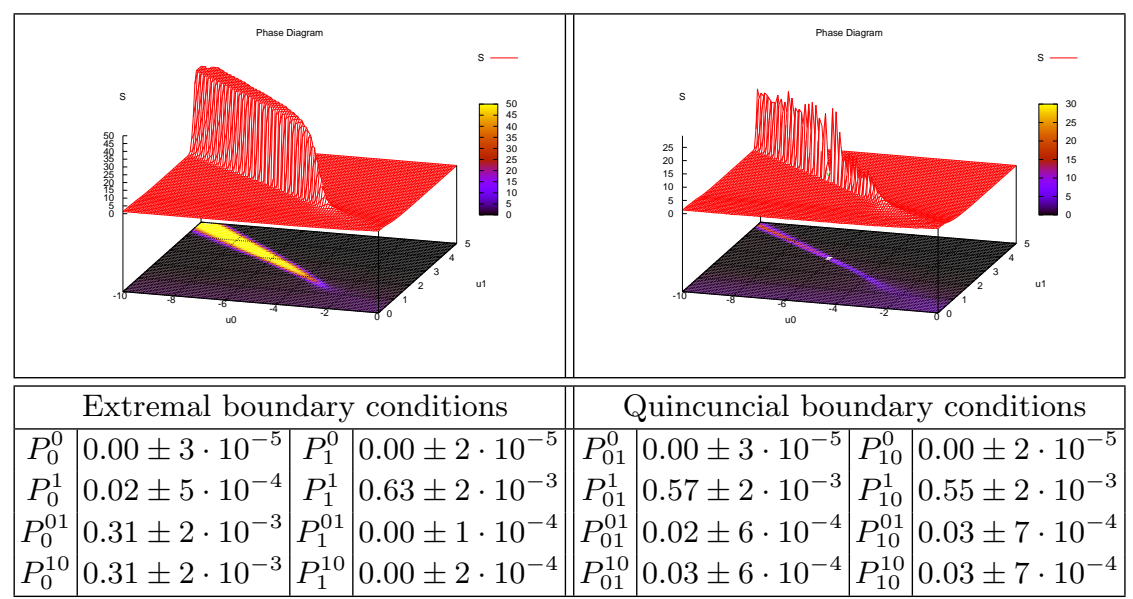

Table 1. Simulation results obtained with attractive Hopfield-like networks presenting the values of the two measures $S_{e}$ and $S_{q}$ and, below, the probabilities (with their $95 \%$ confidence intervals) on the phase transition line $\left(u_{0}+2 \cdot u_{1}=0\right.$ ) to observe specific patterns on the central square.

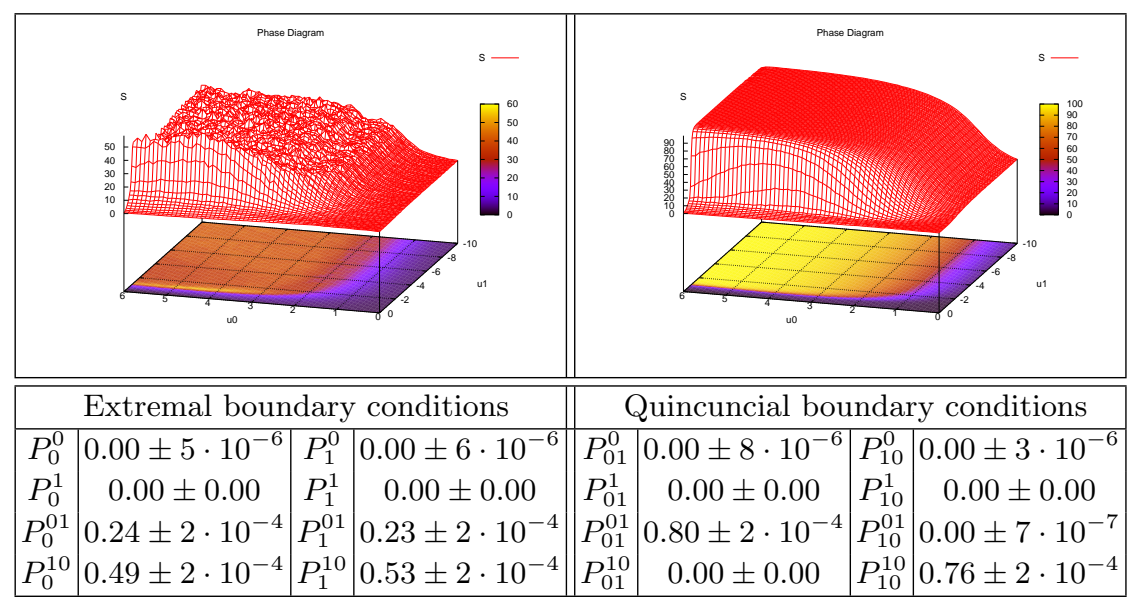

Table 2. Simulation results obtained with repulsive Hopfield-like networks. The reading of these results is the same than in Table 1.

these probabilities, let us give some intuition about the behaviour of such networks on the transition line with extremal and quincuncial boundaries. First, in the case of extremal boundaries, we can think intuitively that the active boundary nodes tends to activate their neighbours. Once active, these neighbours tend to activate their own neighbours and, by induction, tend to propagate the active state until the centre of the network. Thus, an active boundary tends to create an important proportion of patterns $p^{1}$ on the central square. Furthermore, 
the intuition in the case of an inactive boundary is that, since the latter has no influence and since we are on the transition line, no nodes are fixed to an active state and the activating interaction potentials $w_{i j}$ 's in the networks are counter-balanced by the inhibiting interaction potentials $w_{i i}$ 's (autoinhibitions). Consequently, there are only few chances that two neighbour nodes are active together. Thus, the most probable patterns are the quincuncial ones $p^{01}$ and $p^{10}$. Eventually, quincuncial boundaries should create an important proportion of patterns $p^{1}$ since the fixed active nodes tend to activate their neighbourhood in particular for important values of $u_{1}$. Let us remark that all these intuitions are confirmed by the computed probabilities (see Table 1).

To conclude on attractive networks, let us note that: $(i)$ in the quincuncial boundaries case, the minimal probability $\mathcal{P}_{q}$ for which significant values of the measure $S_{q}$ can be observed is $\mathcal{P}_{q}=1-(0.55+0.02+0.03)=0.40 ;($ ii $)$ in the case of extremal boundaries, $\mathcal{P}_{e}$ is equal to $1-(0.02)=0.98$.

Consequently, in attractive networks, on the phase transition line, an event at which $S_{e}$ is incremented has at least $\frac{0.98}{0.40} \approx 2.45$ times more chances to occur than an event at which $S_{q}$ is incremented.

In the case of repulsive networks, the same kind of phenomenon appears. Indeed, the "parabolic" phase transitions (see [13]) induced by the influence of boundary conditions do not depend qualitatively on the nature of boundaries: with extremal and quincuncial boundaries, their parametric domain of emergence stay similar. However, here also, significant quantitative differences appear and, contrary to the attractive case, quincuncial boundaries appear to have more impact (see Table 2). Before explaining this phenomenon and because the behaviour of repulsive networks are less intuitive, let us show why quincuncial boundaries tend to lead their own pattern until the central square of the network.
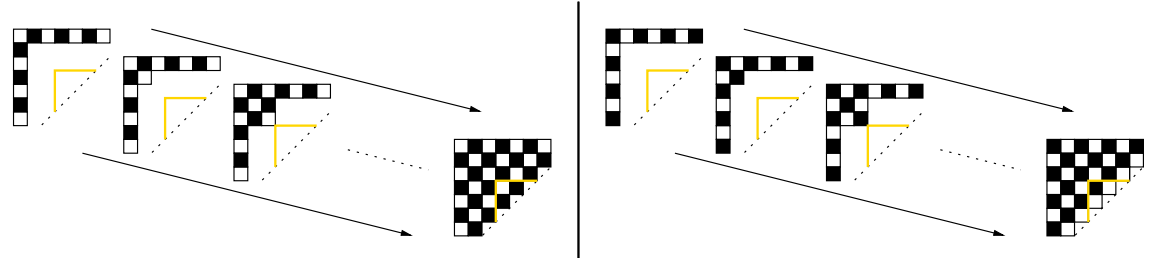

Fig. 1. Construction of a repulsive network with quincuncial boundaries.

Proposition 1. In a repulsive Hopfield-like network represented by a square lattice of odd side size in $\mathbb{Z}^{2}$, quincuncial boundaries tend to reproduce their own pattern the centre of the network.

Proof. The proof is done by induction on a square lattice of odd side size $n$ and by considering the assumption that the quincuncial boundary is $B_{q}^{(01)}$. The aim is to show how to build the most probable configuration being only given the boundary. Let us consider at first time the square $K_{1}^{q}$ of size $2 \times 2$ in the upper 
left corner of the lattice $R \cup \partial R$, where $\partial R$ is the boundary of $R$. As we know the states of boundary nodes, we have to evaluate the state of the node $n_{1}$ located at the bottom right corner of $K_{1}^{q}$. The known nearest neighbours of this node are active (by hypothesis on the nature of the boundary). Since the network is repulsive, these two nodes tend to inhibit $n_{1}$. Let us now consider the square $K_{2}^{q}$ of size $3 \times 3$ in the upper left corner of the lattice for which we know the state of six nodes. Let us focus on the middle right $\left(n_{2}\right)$ and bottom centre $\left(n_{3}\right)$ nodes of $K_{2}^{q}$. Their known nearest neighbours are inactive. Thus, $n_{2}$ and $n_{3}$ tend to be activated by their own activating retroaction potential and, consequently, as it was the case for $K_{1}^{q}$, they tend to inhibit their nearest neighbour $n_{4}$ located at the bottom right corner of $K_{2}^{q}$. By induction, if we build the network of side size $n$ by constructing step by step upper left squares of side size bigger and bigger, one remark that all the inner squares of odd side size whose centre is the centre of the network contain exactly the pattern of the boundary. It is easy to show that the result stays true if we change boundary $B_{q}^{(01)}$ by $B_{q}^{(10)}$ (see Figure 1). Thus, we have the expected result.

The probabilities given in Table 2 show that the most probable central pattern with extremal boundaries is $p^{10}$. Let us now explain these probabilities and show why the extremal boundaries tend to produce the pattern $p^{10}$ on the central square of repulsive networks.

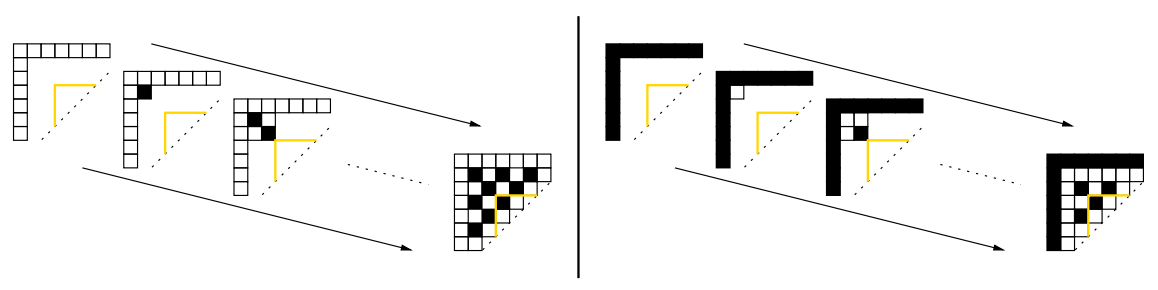

Fig. 2. Construction of a repulsive network with extremal boundaries for important inhibiting interaction potentials.

Proposition 2. In a repulsive Hopfield-like network represented by a square lattice of odd side size in $\mathbb{Z}^{2}$, extremal boundaries tend to reproduce the quincuncial pattern $p^{10}$ until the centre of the network if the inhibiting interaction potentials are greater than the activating retroaction potentials.

Proof. Here also, the proof is performed by induction on a square lattice of odd side size $n$ and by considering extremal boundaries $B_{e}^{(1)}$. Let us perform the proof by building the most probable configuration being only given the boundary. Let us consider the square $K_{1}^{e}$ of size $2 \times 2$ in the upper left corner of the lattice. The node $n_{1}$ at the bottom right corner of $K_{1}^{e}$ is surrounded by two active nodes which tend to inactive $n_{1}$. Let us now consider the square $K_{2}^{e}$ of size $3 \times 3$ in the upper left corner of the lattice. Both the middle right and bottom centre nodes 
$n_{2}$ and $n_{3}$ have one active neighbour. Since we focus on repulsive networks and have done the hypothesis that the inhibiting interaction potentials are sufficiently important, they both tend to be inactivated. Thanks to this hypothesis, it easy to remark that all the neighbours of the boundary nodes are inactivated by the latter. Let us continue with the construction of $K_{2}^{e}$ and consider the node $n_{4}$ located at the bottom right corner of $K_{2}^{e}$. At this step, $n_{4}$ is only surrounded by two inactive nodes. Thus, as no node exerts an inhibiting potential on it, $n_{4}$ has good chance to activate itself thanks to its retroaction potential and will tend to inhibit its other neighbours at next step. By induction, if we build the network of side size $n$ by constructing step by step upper left squares of side size bigger and bigger, one remark that, except the central square of side size $n-2$, all the other central squares of odd side size contain exactly the pattern $p^{10}$. Let us remark that the proof for the extremal boundary $B_{e}^{(0)}$ is captured here (see Figure 2). Consequently, we have the expected result.

This proposition is based on the hypothesis that the inhibiting interaction potentials are greater than the activating retroaction potentials. However, the probabilities obtained by simulations have been computed for the whole phase transition domain in which the values of the parameter $u_{0}$ are greater than those of $u_{1}$ approximatively in 50 percent of the cases. Consequently, it seems that this proposition stay true even without the hypothesis. Besides, we have checked this assumption by computing the probabilities to observe the specific patterns $p^{0}$, $p^{1}, p^{01}$ and $p^{10}$ on the domain of phase transition delimited by $4 \leq u_{0} \leq 5$ and $-2.5 \leq u_{1} \leq-2$. The obtained probabilities are:

\begin{tabular}{|c|c|c|c|}
\hline$P_{0}^{0}$ & $P_{0}^{1}$ & $P_{0}^{01}$ & $P_{0}^{10}$ \\
\hline $0.00 \pm 0.00$ & $0.00 \pm 0.00$ & $0.37 \pm 2 \cdot 10^{-3}$ & $0.52 \pm 2 \cdot 10^{-3}$ \\
\hline$P_{1}^{0}$ & $P_{1}^{1}$ & $P_{1}^{01}$ & $P_{1}^{10}$ \\
\hline $0.00 \pm 0.00$ & $0.00 \pm 0.00$ & $0.28 \pm 2 \cdot 10^{-3}$ & $0.59 \pm 2 \cdot 10^{-3}$ \\
\hline
\end{tabular}

and show that the hypothesis is not necessary for Proposition 2 to be valid.

To conclude on repulsive networks, let us note that: $(i)$ in the extremal boundaries case, the minimal probability $\mathcal{P}_{e}$ for which significant values of the measure $S_{e}$ can be observed is $\mathcal{P}_{e}=1-(0.23+0.49)=0.28 ;(i i)$ in the case of quincuncial boundaries, $\mathcal{P}_{q}$ is equal to 1 .

Consequently, in repulsive networks, on the phase transition line, an event at which $S_{q}$ is incremented has at least $\frac{1}{0.28} \approx 3.57$ times more chances to occur than an event at which $S_{e}$ is incremented.

\section{Architecture of real genetic regulation networks}

In this section, we will show that the previous results on the dependence of core states on the boundary states of the interaction network are also observed in real regulatory networks. We will use for that two simple real genetic networks, the first related to the control of the cell cycle in superior eukaryotes and the second to the regulation of hair morphogenesis in mice. Since we have no 
particular indication about the interaction weights, we fix their absolute values to 1 and the thresholds to 0 and use the deterministic Hopfield-like evolution law.

\subsection{Cell cycle}

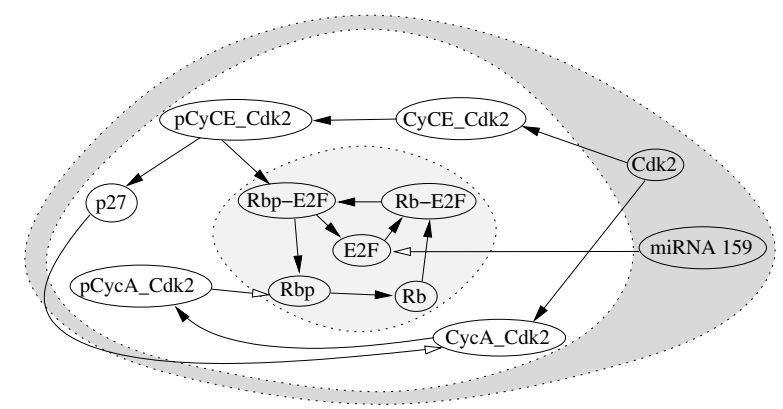

Fig. 3. Genetic regulation network controlling the cell cycle in eukariotes cells (the strongly connected component containing the central node Rbp-E2F is in a light grey space and the boundary node is in a dark grey space).

\begin{tabular}{|c||c|c||c|c|c|}
\hline \multicolumn{1}{|c|}{} & \multicolumn{2}{c||}{ Sequential updating } & \multicolumn{3}{c|}{ Parallel updating } \\
\hline Nature & Attractor & ABRS & Attractor & ABRS & AD \\
\hline \hline Fixed point 1 & 000000000000 & $6.25 \%$ & 000000000000 & $0.5 \%$ & 1.45 \\
\hline Fixed point 2 & 000000011111 & $56.25 \%$ & 000000011111 & $99.5 \%$ & 5.40 \\
\hline \hline Limit cycle 1 & 000000001000 & $37.5 \%$ & None & & \\
& 000000010111 & & & & \\
\hline
\end{tabular}

Table 3. Attractors and attraction basins relative sizes (ABRS) of the cell cycle network dynamics for the sequential and parallel updating iteration modes. The nodes are ordered as follows: p27, Cdk2, pCyCE_Cdk2, CyCE_Cdk2, miRNA 159, pCycA_Cdk2, CycA_Cdk2, Rbp-E2F, Rb-E2F, E2F, Rbp and Rb. The attractor average distance $(\mathrm{AD})$ is the average number of transitions needed from an initial configuration to reach the attractor ; this average distance is correlated with the ABRS.

The genetic network controlling the cell cycle in superior eukariotes cells $[14,15]$ possesses a core made of the gene Rbp-E2F (whose eccentricity is equal to 2) whose strongly connected component is down-regulated in human by one microRNA (miRNA 159 acting on the transcription factor E2F). The frontier of its interaction graph contains the two elements Cdk2 and miRNA 159. For this study, we have focused on the impact of two nodes: the boundary node miRNA 159 because of its direct interaction on the central strongly connected component and the protein p 27 since it has the interesting characteristic to be the node of 
maximal eccentricity $(=7)$ in the interaction graph (see Figure 3). Note that the interaction graph contains only one connected component having at least one (here two) positive circuit of interactions (a circuit is positive if its number of inhibiting edges is even). Hence, from $[16,17,18,19,20,21,22]$, we can expect only $2^{1}=2$ fixed configurations for the network dynamics and an upper bound for this number of $2^{2}$. On Table 3, we see that, if the state of p27 and miRNA 159 are not fixed to particular values, then this number is in reality 2 , plus one (resp. zero) limit cycle in the case of sequential (resp. parallel) updating mode. The conjecture that the number of fixed configurations is equal to $2^{m}$, where $m$ is the number of connex components having at least one positive circuit is here available, as well as the upper bound. The asymptotic result [22] claiming that the number of attractors is of the order of magnitude of the square root of the network size (here $\sqrt{12}$ ) is also verified. If the boundary state of p27 is fixed to 1 , then we keep in the parallel case the two fixed configurations. If the state of miRNA 159 is fixed to 1 , due to its constant inhibition, we observe 6 attractors in the parallel case:

\begin{tabular}{|c|c|c|}
\hline Nature & Attractor & ABRS \\
\hline \hline Fixed point 1 & 000010000000 & $0.12 \%$ \\
\hline Fixed point 2 & 000010011011 & $0.54 \%$ \\
\hline \hline Limit cycle 1 & 000010001010 & $10.6 \%$ \\
& 000010010001 & \\
\hline Limit cycle 2 & 000010010011 & $26.6 \%$ \\
& 000010001011 & \\
& 000010011001 & \\
& 000010011010 & \\
\hline Limit cycle 3 & 000010010010 & $32.4 \%$ \\
& 000010000011 & \\
& 000010001001 & \\
& 000010011000 & \\
\hline Limit cycle 4 & 000010000010 & $23.7 \%$ \\
& 000010000001 & \\
& 000010001000 & \\
& 000010010000 & \\
\hline
\end{tabular}

If we fix both p27 and miRNA 159 to 1 , we keep the previous attractors.

\subsection{Mice hair}

The genetic network controlling the hair morphogenesis in mice [23] has a core containing 2 genes, $\beta$-catenin and Cyclin D1 (whose eccentricity is equal to 5 , see Figure 4) with a motif called incoherent feed-forward [24]. Its boundary is composed by the five following nodes: Smad3, miRNA 141, EphA3, SrC and Zfhx3. In the following, we are going to focus on the influence induced by the two boundary nodes of maximal eccentricity $(=9)$ which have two different actions on the network. Indeed, the strongly connected component containing the core 


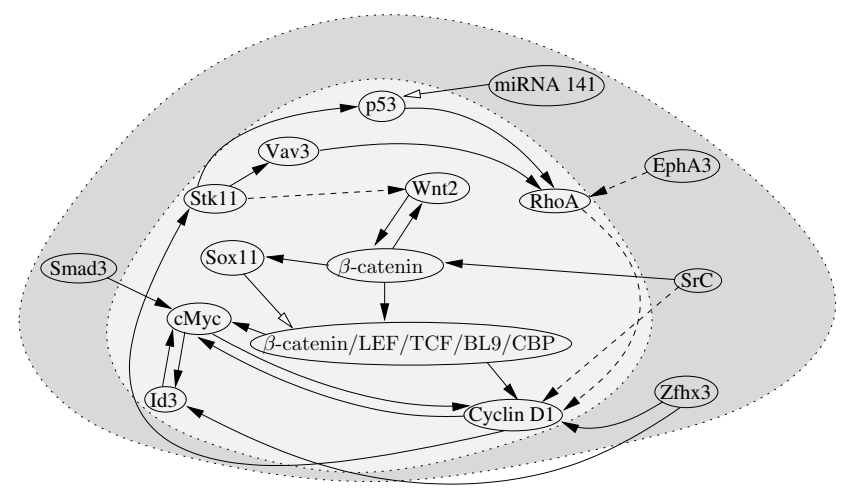

Fig. 4. Genetic regulation network controlling the hair morphogenesis in mice (the strongly connected component containing the central nodes is in a light grey space and the boundary nodes are in a dark grey space).

\begin{tabular}{|c||c|c||c|c|c|}
\hline \multicolumn{1}{|c||}{} & \multicolumn{2}{c||}{ Sequential updating } & \multicolumn{3}{c|}{ Parallel updating } \\
\hline Nature & Attractor & ABRS & Attractor & ABRS & AD \\
\hline \hline Fixed point 1 & 0000000000000000 & $1.56 \%$ & 0000000000000000 & $\approx 0.00 \%$ & 0.75 \\
\hline Fixed point 2 & 0011111001101110 & $96.88 \%$ & 0011111001101110 & $99.66 \%$ & 4.14 \\
\hline Fixed point 3 & 0000010000001100 & $1.56 \%$ & 0000010000001100 & $\approx 0.00 \%$ & 0.5 \\
\hline \hline Limit cycle 1 & None & - & 0011010001101100 & $0.34 \%$ & 2.45 \\
& & & 0000111000001110 & & \\
\hline
\end{tabular}

Table 4. Attractors and attraction basins relative sizes (ABRS) of the dynamics of the network modelling the hair morphogenesis of mice for the sequential and parallel updating iteration modes. The nodes are ordered as follows: miRNA 141, EphA3, p53, Vav3, Stk11, Wnt2, RhoA, Smad3, SrC, Id3, Cyclin D1, Zfhx3, Sox11, $\beta$-catenin, cMyc and $\beta$-catenin/LEF/TCF/BL9/CBP.

is down-regulated in human by the micro-RNA 141 [25,26], acting on the protein $\mathrm{p} 53$, and up-regulated by the protein phosphatase EphA3. The interaction graph contains only one connected component having at least one (here five) positive circuits of interactions, as in the previous example of the cell cycle, we expect only $2^{1}=2$ fixed configurations for the network dynamics and an upper bound of $2^{5}$. On Table 4, we see that, if the boundary state of miRNA 141 is not fixed to a specific value, then this number is in reality 3 , plus one limit cycle in the case of parallel updating mode, which disappears by introducing delays in the graph for the indirect interactions (arrows in dashed lines on Figure 4) for which we introduce each time an intermediary node (i.e. one node between Stk11 and Wnt2, one between RhoA and Cyclin D1 and one between SrC and Cyclin D1). The conjecture introduced above is false in this case but the upper bound stays correct. The asymptotic (in the number of genes) result claiming that the number of attractors is of the order of magnitude of the square root of the network size (here $\sqrt{16}$ ) is also verified. If the boundary state of miRNA 141 
is equal to 1 , then we keep in the parallel case the same attractors by chanching the state of miRNA 141 from 0 to 1 (we only have the state of p53 constantly equal to 0 due to the inhibition), conserved (except the limit cycle) by adding delays. On the contrary, by changing the state of EphA3 from 0 to 1, we have only one fixed configuration in the parallel case: 0111111001101110, conserved by adding delays.

\section{Conclusion}

We have studied, in this paper, the influence of boundaries on Hopfield-like regulatory networks: this influence is present in case of quincuncial boundaries for neural networks especially when the interactions are repulsive. The sensitivity in real genetic networks appears also dominant in the case of inhibitory actions exerted by micro-RNAs. A more systematic study could be processed in the future in order to confirm this dominant influence of negative interactions, for which the Hopfield-like regulatory networks seem to be less robust than for the positive ones (activations).

\section{References}

1. Maslov, S., Sneppen, K., Eriksen, K.A., Yan, K.K.: Upstream Plasticity and Downstream Robustness in Evolution of Molecular Networks. BMC Evolutionary Biology 4(9) (2004)

2. Handorf, T., Ebenhöh, O., Heinrich, R.: Expanding Metabolic Networks: Scopes of Compounds, Robustness, and Evolution. Journal of Molecular Evolution 61(4) (2005) 498-512

3. Kitano, H.: Towards a Theory of Biological Robustness. Molecular Systems Biology 3 (2007)

4. Kitano, H.: Biological Robustness in Complex Host-Pathogen Systems. Progress in Drug Research 64(239) (2007) 241-263

5. Demongeot, J., Morvan, M., Sené, S.: Impact of Fixed Boundary Conditions on the Basins of Attraction in the Flower's Morphogenesis of Arabidopsis Thaliana. In: Proceedings of the 22nd International Conference on Advanced Information Networking and Applications Workshops, IEEE Computer Society Press (2008) $782-789$

6. Demongeot, J., Morvan, M., Sené, S.: Robustness of Dynamical Systems Attraction Basins Against State Perturbations: Theoretical Protocol and Application in Systems Biology. In: Proceedings of the 2nd International Conference on Complex Intelligent and Software Intensive Systems, IEEE Computer Society Press (2008) 675-681

7. Hopfield, J.J.: Neural Networks and Physical Systems with Emergent Collective Computational Abilities. Proceedings of the National Academy of Sciences of the United States of America 79 (1982) 2554-2558

8. Albert, R., Barabási, A.L.: Statistical Mechanics of Complex Networks. Reviews of Modern Physics 74 (2002) 47-97

9. Barabási, A.L., Oltvai, Z.N.: Network Biology: Understanding the Cell's Functional Organization. Nature Reviews Genetics 5 (2004) 101-113 
10. Wolfram, S.: Statistical mechanics of cellular automata. Reviews of Modern Physics 55 (1983) 601-644

11. Ayoubi, R.A., Ziade, H.A., Bayoumi, M.A.: Fault Tolerant Hopfield Associative Memory on Torus. In: Proceedings of the 18th IEEE International Symposium on Defect and Fault Tolerance in VLSI Systems, IEEE Computer Society Press (2003) 369 (8 pages)

12. Regnault, D., Schabanel, N., Thierry, E.: Progresses in the Analysis of Stochastic 2D Cellular Automata: A Study of Asynchronous 2D Minority. In: Proceedings of the International Symposium on Mathematical Foundations of Computer Science. Volume 4708 of Lecture Notes in Computer Science., Springer (2007) 320-332

13. Demongeot, J., Sené, S.: Boundary Conditions and Phase Transitions in Neural Networks. Simulation Results. To appear in Neural Networks (2008)

14. Withfield, M.L., Sherlock, G., Saldanha, A.J., Murray, J.I., Ball, C.A., Alexander, K.E., Matese, C., Perou, C.M., Hurt, M.M., Botstein, D.: Identification of Genes Periodically Expressed in the Human Cell Cycle and Their Expression in Tumors. Molecular Biology of the Cell 13 (2002) 1977-2000

15. Abacci, A.: A Modelisation of the CCMRN in Superior Eukaryotes Cell Population. Master's thesis, University Joseph Fourier of Grenoble (2006)

16. Demongeot, J., J., A., Thuderoz, F., Baum, T.P., Cohen, O.: Genetic Regulation Networks: Circuits, Regulons and Attractors. Comptes Rendus Biologies 326 (2003) 171-188

17. Demongeot, J., Thuderoz, F., P., B.T., Berger, F., Cohen, O.: Bio-array Images Processing and Genetic Networks Modelling. Comptes Rendus Biologies 326 (2003) $487-500$

18. Aracena, J., Ben Lamine, S., Mermet, M.A., Cohen, O., Demongeot, J.: Mathematical Modelling in Genetic Networks: Relationships Between the Genetic Expression and Both Chromosomic Breakage and Positive Circuits. IEEE Transactions in Systems Man Cybernetics 3 (2003) 825-834

19. Aracena, J., Demongeot, J., Goles, E.: Mathematical Modelling in Genetic Networks. IEEE Transaction in Neural Networks 15 (2004) 77-83

20. Aracena, J., Demongeot, J., Goles, E.: Fixed Points and Maximal Independent Sets on AND-OR Networks. Discrete Applied Mathematics 138 (2004) 277-288

21. Aracena, J., Demongeot, J., Goles, E.: On Limit Cycles of Monotone Functions With Symmetric Connection Graphs. Theoretical Computer Science 322 (2004) $237-244$

22. Aracena, J., Demongeot, J.: Mathematical Methods for Inferring Regulatory Networks Interactions: Application to Genetic Regulation. Acta Biotheoretica 51 (2004) 391-400

23. Michon, F., Forest, L., Collomb, E., Demongeot, J., Dhouailly, D.: BMP-2 and BMP-7 Play Antagonistic Roles in Feather Induction. Development (2008) In press.

24. Elena, A., Demongeot, J.: Interaction Motifs in Regulatory Networks and Structural Robustness. In: Proceeding of the International Conference on Complex, Intelligent and Software Intensive Systems, IEEE Computer Society Press (2008) $682-686$

25. Demongeot, J., Moreira, A.: A Circular RNA at the Origin of Life. Journal of Theoretical Biology 249 (2007) 314-324

26. Demongeot, J., Moreira, A.: A Circular Hamming Distance, Circular Gumbel Distribution, RNA Relics and Primitive Genome. In: Proceedings of the International Conference on Advanced Information Networking and Applications Workshops, IEEE Computer Society Press (2007) 719-726 